Proceedings of the International School and Conference on Optics and Optical Materials, ISCOM07, Belgrade, Serbia, September 3-7, 2007

\title{
Imperfect Cloaking Devices Based on Metamaterials
}

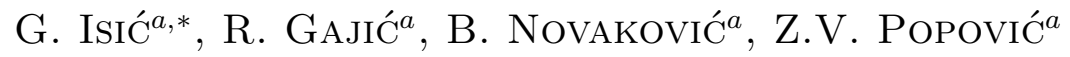 \\ AND K. HINGERL ${ }^{b}$ \\ ${ }^{a}$ Institute of Physics, Pregrevica 118, P.O. Box 68, 11080 Belgrade, Serbia \\ ${ }^{b}$ Christian Doppler Labor, Institut für Halbleiter- und Festkörperphysik \\ Universität Linz, A 69, 4040 Linz, Austria
}

\begin{abstract}
Cloaking devices designed using the coordinate transform approach were shown to be realizable, at least in principle, within the realm of electromagnetic metamaterials. In this paper we investigate the strictness of conditions imposed on the parameters of metamaterial cloaks by calculating the degree of wave scattering when those parameters have variations with respect to theoretically ideal values. A simple idea is used to obtain analytic results for the case of the nonideal two-dimensional cloaking cylinder. Also, results of realistic finite element simulations of the Helmholtz equation are presented and it is found that they are in excellent agreement with the analytic results.
\end{abstract}

PACS numbers: 41.20.Jb, 42.25.Fx, 78.20.Ci

\section{Introduction}

The advent of metamaterials over the past several years has refueled the interest in electromagnetics for various structures having unusual values of $\varepsilon$ and $\mu$. Negative refraction [1], superlensing [2], and electromagnetic cloaking [3-6] are only few of the many exciting phenomena expected within the domain of metamaterials [7].

The idea of cloaking devices based on the coordinate transform design, recently suggested in [3], has been shown to be the most versatile one amongst several other invisibility ideas [6, 8-10]. The recent article [11] from Leonhardt is a beautiful demonstration of how the remarkable phenomena associated with metamaterials can be unified within the framework of general relativity.

So far, most of attention regarding these cloaking devices has been given to 2D structures, since they are much easier to manufacture than 3D ones. However,

*corresponding author; e-mail: isicg@phy.bg.ac.yu 
in the $2 \mathrm{D}$ case, the theory suggests that the material parameters of the cloaking shell should have singular values on their inner surface. Our intention in this paper is to investigate what happens when finite values of the material parameters are used.

\section{The imperfect cloaking device}

We consider an infinite 2D cloaking cylinder [4]. Cylindrical coordinates are employed for both the "physical" space, $(r, \varphi, z)$, and the "transformation"space, $\left(\rho, \varphi^{\prime}, z^{\prime}\right)$. The "physical" space is where we have the actual metamaterial cloak with permittivity and permeability tensors $\boldsymbol{\varepsilon}$ and $\boldsymbol{\mu}$, while in the "transformation" space the transformed material parameters labeled $\varepsilon^{\prime}$ and $\boldsymbol{\mu}^{\prime}$ are obtained. In case of ideal cloak, here should be only vacuum, i.e. the ideal cloak has $\varepsilon^{\prime}=1$ and $\boldsymbol{\mu}^{\prime}=1$. The recipe for obtaining $\varepsilon^{\prime}$ and $\boldsymbol{\mu}^{\prime}$ from $\boldsymbol{\varepsilon}$ and $\boldsymbol{\mu}$ (or vice versa) when the mapping between the "physical" and "transformation" space is defined, is discussed in $[3,11,12]$.

The structure to be considered consists of the cloaking device, occupying the volume $R_{1}<r<R_{2}$, and the cloaked object which is inside $r<R_{1}$. We consider the mapping from the "transformation" space defined by $r=g(\rho), \varphi=\varphi^{\prime}$ and $z=z^{\prime}$ with $g(\rho)$ given by

$$
\begin{aligned}
& g(\rho)=\rho R_{1} / \rho_{1}, \quad \text { for } \quad \rho<\rho_{1}, \\
& g(\rho)=\alpha \rho+\beta, \quad \text { for } \quad \rho_{1} \leq \rho<R_{2}, \\
& g(\rho)=r, \quad \text { for } \quad \rho>R_{2},
\end{aligned}
$$

with $\alpha=\left(R_{2}-R_{1}\right) /\left(R_{2}-\rho_{1}\right)$ and $\beta=R_{2}\left(R_{1}-\rho_{1}\right) /\left(R_{2}-\rho_{1}\right)$. Clearly, the case with $\rho_{1}=0$, is the case of the ideal cloak [4] having the relative permittivity and permeability given by

$$
\begin{aligned}
& \mu_{r}^{\mathrm{Id}}=\left(r-R_{1}\right) / r, \quad \mu_{\varphi}^{\mathrm{Id}}=1 / \mu_{r}^{\mathrm{Id}}, \\
& \mu_{z}^{\mathrm{Id}}=\mu_{r}^{\mathrm{Id}} / \alpha^{\mathrm{Id}}, \quad \alpha^{\mathrm{Id}}=\left(R_{2}-R_{1}\right) / R_{2}
\end{aligned}
$$

with $\varepsilon=\boldsymbol{\mu}$. The problem with these parameters is that they all have singular values at the boundary $r=R_{1}$, meaning that the value of $\mu_{r}^{\mathrm{Id}}$ has to be precisely zero. To analyse an imperfect cloak we assume that the deviations have precisely the values corresponding to the case of finite $\rho_{1}$. The parameters of the cloak are then shown to be given by

$$
\mu_{r}=(r-\beta) / r, \quad \mu_{\varphi}=1 / \mu_{r}, \quad \mu_{z}=\mu_{r} / \alpha^{2},
$$

and there are no singular material parameters since $\beta<R_{1}$. For a given cloaking device, this procedure will not give exact solutions for the scattering, but it allows for a fairly good estimate of the cloaking performance if the expected deviations are known in advance, e.g. if $\delta \mu_{r}=\mu_{r}-\mu_{r}^{\text {Id }}$ is taken to be representative, we put

$$
\mu_{r}\left(r=R_{1}\right)=\delta \mu_{r} \rightarrow \rho_{1}=\frac{R_{1} R_{2} \delta \mu_{r}}{R_{2}-R_{1}\left(1-\delta \mu_{r}\right)}
$$

The solution for the scattering on such a cloaking device is easily obtained in the "transformation" domain where the material properties for $\rho<\rho_{1}$ are given by 


$$
\begin{aligned}
& \varepsilon_{r}^{\prime}=\varepsilon, \quad \mu_{r}^{\prime}=\mu, \quad \varepsilon_{\varphi}^{\prime}=\varepsilon, \quad \mu_{\varphi}^{\prime}=\mu, \quad \varepsilon_{z}^{\prime}=\gamma^{2} \varepsilon, \quad \mu_{z}^{\prime}=\gamma^{2} \mu, \\
& \gamma=R_{1} / \rho_{1},
\end{aligned}
$$

where we have assumed, for the sake of simplicity, that the cloaked object is isotropic with parameters $\varepsilon$ and $\mu$. Outside the volume, i.e. for $\rho>\rho_{1}$, in the "transformation" space, there is only vacuum, since we assume that the cloak material conditions are exactly met in the "physical" space.

In the present case, a 2D problem with diagonal $\boldsymbol{\varepsilon}^{\prime}$ and $\boldsymbol{\mu}^{\prime}$, the solutions for the wave equation are split to transverse electric (TE) waves with the electric field along the $z$-axis, and transverse magnetic (TM) waves, with the magnetic field along the $z$-axis. Since any result for the TE waves can be converted to the corresponding result for the TM waves, with the change $\boldsymbol{E} \leftrightarrow \boldsymbol{H}$ and $\boldsymbol{\varepsilon} \leftrightarrow-\boldsymbol{\mu}$, we shall consider only TE waves. We label the $z$-component of the electric field of the TE wave in the "transformation" space with $\bar{E}_{z}$, so the TE Helmholtz equation $(\boldsymbol{E}(t) \sim \exp (-\mathrm{i} \omega t)$ is assumed $)$ reads

$$
\frac{1}{\rho} \frac{\partial}{\partial \rho}\left(\rho \frac{\partial \bar{E}_{z}}{\partial \rho}\right)+\frac{1}{\rho^{2}} \frac{\partial^{2} \bar{E}_{z}}{\partial \varphi^{\prime 2}}+k^{2} \bar{E}_{z}=0,
$$

where $k^{2}=k_{\mathrm{c}}^{2}=\mu \varepsilon \gamma^{2} k_{0}^{2}$, for $\rho<\rho_{1}$, and $k^{2}=k_{0}^{2}$, for $\rho>\rho_{1}$.

The problem of plane wave scattering on an infinite cylinder is a standard one in the scattering theory [13]. Our approach is valid for any type of the hidden object, however to keep it short, we assume that the hidden object is a perfect electric conductor (for a more detailed analysis see [14]), allowing for simpler results. It is evident that the cloaking shell effectively shrinks our metallic cylinder to the cylinder with the same parameters but with smaller radius, i.e. from $R_{1}$ in the "physical" space to $\rho_{1}$ in the transformation space. Therefore, the scattering field, $\bar{E}_{z}^{\mathrm{s}}$, in the "transformation" space is given by

$$
\begin{aligned}
& \bar{E}_{z}^{\mathrm{s}}=E_{0} \sum_{n=-\infty}^{\infty} \exp (\mathrm{i} n \tilde{\varphi}) a_{n} H_{n}^{(1)}\left(k_{0} \rho\right), \\
& \tilde{\varphi}=\varphi-\varphi_{k}+\pi / 2, \quad n=0, \pm 1, \pm 2, \ldots .
\end{aligned}
$$

where $\varphi_{k}$ is the direction of the incident plane wave with respect to $x$-axis, $H_{n}^{(1)}$ is the Hankel function of the first kind and the scattering coefficients, $a_{n}$, are given by

$$
\mathrm{TE}: \quad a_{n}^{\mathrm{TE}}=-\frac{J_{n}\left(k_{0} \rho_{1}\right)}{H_{n}^{(1)}\left(k_{0} \rho_{1}\right)}, \quad \mathrm{TM}: \quad a_{n}^{\mathrm{TM}}=\frac{J_{n+1}\left(k_{0} \rho_{1}\right)-J_{n-1}\left(k_{0} \rho_{1}\right)}{H_{n-1}\left(k_{0} \rho_{1}\right)-H_{n+1}\left(k_{0} \rho_{1}\right)} .
$$

The solution for $\bar{E}_{z}^{\mathrm{s}}$ in the "transformation" space can be transformed to obtain the solution in the "physical" space. We are interested mostly in the fields outside the cloak, $\rho>R_{2}$, and here the fields in both spaces are exactly the same. Having established the solutions for the imperfect cloak, which has non-singular values for the material parameters, we now investigate the $\rho_{1} \rightarrow 0$ limit. In this case, the scattering coefficients, $a_{n}$, are given as 


$$
a_{0}^{\mathrm{TE}} \rightarrow \frac{\mathrm{i} \pi}{2 \ln \left(k_{0} \rho_{1}\right)} \text { and } a_{n}^{\mathrm{TE}} \rightarrow-\frac{\mathrm{i} \pi}{2^{2 n} n !(n-1) !}\left(k_{0} \rho_{1}\right)^{2 n}, \quad n \geq 1
$$

for TE waves, and

$$
a_{0}^{\mathrm{TM}} \rightarrow \frac{\mathrm{i} \pi}{4}\left(k_{0} \rho_{1}\right)^{2} \text { and } a_{n}^{\mathrm{TM}} \rightarrow \frac{\mathrm{i} \pi}{2^{2 n} n !(n-1) !}\left(k_{0} \rho_{1}\right)^{2 n}, \quad n \geq 1,
$$

for TM waves. Hence, each of the scattering coefficients $a_{n}$ decays and our imperfect cloak approaches the ideal one. From the equations above, we see that the convergence of the zeroth order scattered TE cylindrical wave on the hidden ideal electrical conductor is by far the slowest which explains the isotropic scattering on the cloaking device mentioned in [4].

\section{Numerical results}

To illustrate the performance of an imperfect cloak, we shall take that $\lambda_{0}=0.25$ (these are arbitrary units, e.g. meters), $R_{1}=\lambda_{0}, R_{2}=2 \lambda_{0}$, assume that the imperfection of the cloak parameters is determined by $\delta \mu_{r}$ at $r=R_{1}$, as described in the previous section, and take that the hidden object is an ideal electric conductor. Then, we quantify the quality of the cloak, $q$, with the factor by which the actual scattering width, $d_{\mathrm{s}}^{0}$, of the hidden object is decreased, $q=d_{\mathrm{s}}^{0} / d_{\mathrm{s}}$ with $d_{\mathrm{s}}$ being the scattering width with the cloak. The scattering width, defined as the ratio of the scattered flux and the incoming power flux density (per length) for the cylinder without the cloak is found to be $d_{\mathrm{s}}^{0} \approx 1.145$. When the cloak is present, the $a_{0}$ scattering coefficient dominates, which is confirmed by our finite element (FE) simulations given below, so we have

$$
q \approx 2.29 \ln ^{2}\left(k_{0} \rho_{1}\left(\delta \mu_{r}\right)\right) / \pi \lambda_{0}
$$

From (4) we obtain that in order to achieve an electromagnetic "shrinking" by 10 , i.e. $q=10$, we need to control the material parameter $\delta \mu_{r}$ within approximately 0.01 . Having in mind that values of $\varepsilon$ and $\mu$ below unity imply superluminal
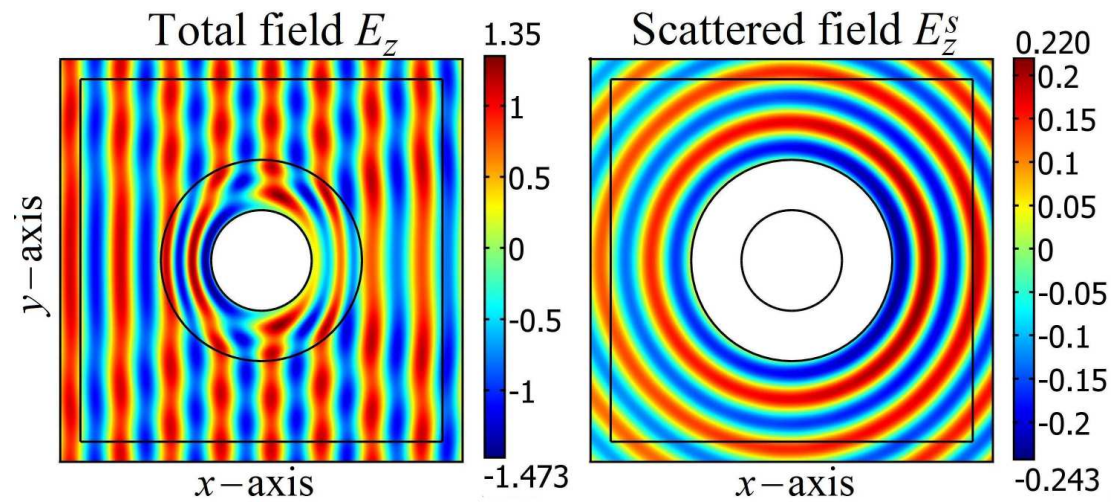

Fig. 1. FE simulation results. Smaller circle is the inner boundary of the cloak, $R_{1}=$ $\lambda_{0}=0.25$, and the bigger circle is the external cloak boundary $R_{2}=2 \lambda_{0}$. The simulation was carried out for $\rho_{1}=0.02$. 


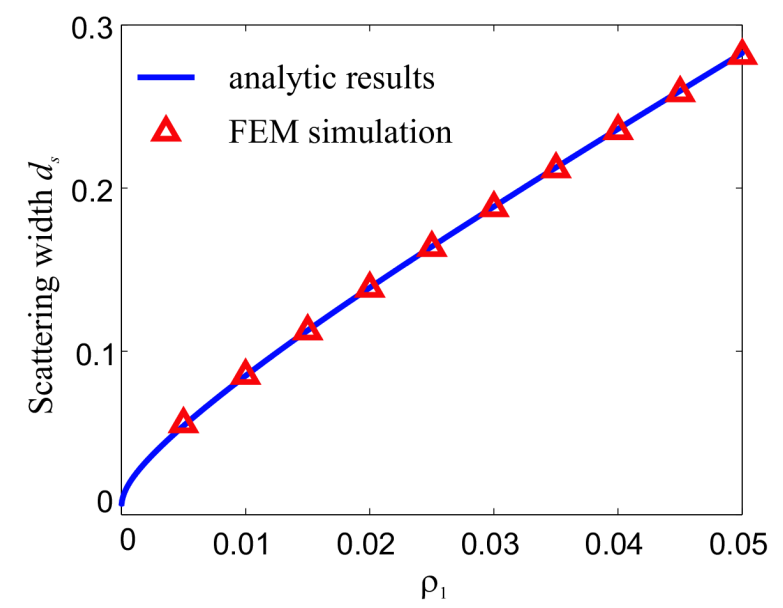

Fig. 2. Dependence of $d_{\mathrm{s}}$ on $\rho_{1}$, comparison of analytic and FE results.

phase velocities and, thus, strong material dispersion, such stringent conditions on $\varepsilon$ and $\mu$ as indicated above, mean that the band width within which the cloaking is effective is going to be very narrow (which is easily estimated given the particular dispersion formula). Also, since $\ln ^{2}\left(k_{0} \rho_{1}\right)$ goes to infinity when $\delta \mu_{r} \rightarrow 0$, the curve $q\left(\delta \mu_{r}\right)$ is very steep for $q>10$, so even a small increase in $\delta \mu_{r}$ will significantly decrease the value of $q$.

Figure 1 shows the scattered field obtained by our FE simulations for the case of cloak with $\rho_{1}=0.02$ and incident TE wave. The square drawn inside the simulation domain, represents the contour along which the power flux density was integrated to obtain the FE result for the scattering width. The results were compared to the values of $d_{\mathrm{s}}$ calculated analytically, and this is shown in Fig. 2.

The mesh element size used in FE simulations was around $\lambda / 10$, meaning that the simulated structure roughly corresponds to a metamaterial with the unit cell size of $\lambda / 10$, which is a typical value. The good agreement with FE simulations confirms our assumption that most of the scattering comes from the part of cloak with $r \approx R_{1}$.

\section{Conclusion}

Simple analytic results for estimating the cloaking performance (scattered field and scattering width) are presented. On the example of a thin cylinder made of ideal electrical conductor we have shown that the best it can be expected from a realistic cloaking shell is that it decreases its scattering width by around one order of magnitude and it is for the shell of thickness comparable to the radius of the concealed object. 


\section{Acknowledgments}

This work is supported by the Serbian Ministry of Sicence project 141047. K.H. is grateful for partial support from European Community Project N2T2. We are also grateful to Photeon and Heinz Syringer from Photeon Technologies for financial support and Johann Messner from the Linz Supercomputer Center for technical support.

\section{References}

[1] U. Leonhardt, T.G. Philbin, New J. Phys. 8, 247 (2006).

[2] J.B. Pendry, Phys. Rev. Lett. 85, 3966 (2000).

[3] J.B. Pendry, D. Schurig, D.R. Smith, Science 312, 1780 (2006).

[4] S.A. Cummer, B.I. Popa, D. Schurig, D.R. Smith, J.B. Pendry, Phys. Rev. E 74, 036621 (2006).

[5] D. Schurig, J.J. Mock, B.J. Justice, S.A. Cummer, J.B. Pendry, A.F. Starr, D.R. Smith, Science 314, 977 (2006).

[6] U. Leonhardt, Science 312, 1777 (2006).

[7] C. Caloz, T. Itoh, Electromagnetic Metamaterials: Transmission Line Theory and Microwave Applications. The Engineering Approach, Wiley, New Jersey 2006.

[8] A. Alu, N. Engheta, Phys. Rev. E 72, 016623 (2005).

[9] G.W. Milton, N.-A.P. Nicorovici, Proc. R. Soc. A 462, 3027 (2006).

[10] D.A.B. Miller, Opt. Express 14, 12457 (2006).

[11] U. Leonhardt, New J. Phys. 8, 118 (2006).

[12] A.J. Ward, J.B. Pendry, J. Mod. Opt. 43, 773 (1996).

[13] L. Tsang, J.A. Kong, K.-H. Ding, Scattering of Electromagnetic Waves: Theories and Applications, Wiley, New York 2000, p. 41.

[14] G. Isić, R. Gajić, B. Novaković, Z.V. Popović, K. Hinger, submitted to Opt. Exp.. 